\title{
Canadian content at GU-ASCO 2014: Highlights of research involving Canadian researchers
}

Cite as: Can Urol Assoc J 2014;8(3-4Suppl2):S16-8. hitrp://dx.doi.org/10.5489/cuai.2015

Published online April 14, 2014.

\section{Abstract}

The 2014 Genitourinary Cancers Symposium (GU-ASCO 2014) provided an opportunity for researchers from around the world to present their research in a variety of fields, including prostate, renal cell, penile, urethral and testicular cancers. Over the symposium's 3 days, Canadian researchers were well-represented, with a number of oral abstract podium presentations and many more research posters. The following section provides brief summaries of some of the most interesting work involving Canadians presented at GU-ASCO 2014 and a listing of all the studies that included contributions from Canadian researchers.

\section{Canadian highlights in prostate cancer research at GU-ASCO 2014}

\section{Effect of prior abiraterone on enzalutamide activity in metastatic castrate- resistant prostate cancer}

Dr. Arun Azad (British Columbia Cancer Agency, Vancouver) was part of the research team investigating the impact of prior abiraterone therapy on the activity of enzalutamide in men with metastatic castrate-resistant prostate cancer (mCRPC). ${ }^{1}$ In this study, the investigators retrospectively reviewed the records of 195 patients treated with enzalutamide between January 2009 and August 2013. They evaluate the association between prior abiraterone therapy and the rates of $30 \%$ or greater prostatespecific antigen (PSA) decline (PSA30). They reported that, overall, $42 \%$ of enzalutamide-treated patients achieved a PSA30; the rates were $39 \%$ among those patients with prior abiraterone treatment and 55\% among abiraterone-naïve patients. The odds of achieving a PSA30 on enzalutamide were 2.3 times higher for abiraterone-naïve patients compared to abiraterone-treated patients (95\% confidence interval $[\mathrm{Cl}] 1.0$ to $5.5, p=0.06)$ after adjusting for prior docetaxel and concurrent steroid use.

\section{Novel prognostic marker for abiraterone-treated patients}

Dr. Azad was also the lead author of a study investigating the utility of a prognostic model (previously developed using data from the Abiraterone Acetate in Castration-Resistant Prostate Cancer Previously Treated With Docetaxel-Based Chemotherapy [COU-AA-301] trial) in an unselected, population-based cohort of patients treated with abiraterone. ${ }^{2}$ The 415 patients included in the analysis were identified from registries at 3 Canadian centres: British Columbia Cancer Agency (Vancouver), Tom Baker Cancer Centre (Calgary) and Princess Margaret Cancer Centre (Toronto). The risk factors included in the prognostic model are: Eastern Cooperative Oncology Group (ECOG) performance status (PS) 2, presence of visceral metastases, time from start of luteinizing-hormone-releasing hormone $(\mathrm{LHRH})$ agonists to start of abiraterone 36 months, low albumin, high alkaline phosphatase (ALP) and high lactate dehydrogenase (LDH). In this Canadian cohort, $21 \%, 50 \%$ and $28 \%$ were classified into good (0-1 risk factors), intermediate (2-3 risk factors) and poor (4-6 risk factors) prognosis, respectively. The median overall survival (OS) durations for these groups were 23.9 months for good prognosis, 17.6 months for intermediate and 8.4 months for poor (statistically-significant differences for intermediate and for poor compared to good prognosis). The investigators concluded that their analysis validates the COU-AA-301 model as a tool for prognostic stratification of $\mathrm{MCRPC}$ patients treated with abiraterone.

\section{Nibrin (NBN) as a predictor of biochemical relapse}

Dr. Alejandro Berlin (Princess Margaret Cancer Centre, Toronto) and colleagues studied a series of DNA damage response (DDR) genes in an effort to determine whether copy number alterations (CNAs) in DDR genes are predictive or prognostic following local treatment for prostate cancer. ${ }^{3}$ The subjects in their study were 126 patients with intermediate-risk prostate cancer who underwent image-guided radiotherapy (IGRT). The investigators reported that NBN gain was a significant independent predictor of five-year biochemical-relapse-free rate following IGRT (48.6\% vs. $78.8 \%$; hazard ratio [HR] 3.14, 95\% Cl 1.42 to $6.94, p=0.004)$. They concluded that, "NBN gain could be the first prostate cancer predictive biomarker to facilitate local treatment decisions using precision medicine approaches."

\section{Adjuvant radiotherapy for highly aggressive disease}

Drs. Giorgio Gandaglia, Paul Perotte, Maxine Sun and Pierre Karakiewicz (Montreal) were part of an international group investigating the effect of adjuvant radiotherapy (aRT) on cancer- 
specific mortality (CSM) after radical prostatectomy (RP). ${ }^{4}$ The investigators analyzed data from 7616 patients with pT3/4 N0/1 prostate cancer treated with RP between 1995 and 2009 in the American Surveillance Epidemiology and End Results (SEER) Medicare-linked database. They tested the effect of aRT on CSM according to a risk score based on the presence or absence of three adverse pathological characteristics (Gleason score 8-10; pT3b/4, lymph node invasion [LNI]).

The researchers reported that aRT was associated with significantly lower 10-year CSM only among patients with 2 or more risk factors (10-year mortality $6.9 \%$ for aRT in this group, compared to $16.2 \%$ for those not receiving aRT, $p=0.002$ ). Among those with a risk score of 0 or 1 , aRT was not associated with a reduction in CSM rate.

\section{Surgical margin status for robotic versus open RP}

Drs. Gandaglia, Sun and Karakiewicz were also part of an international group examining surgical margin status for robotic versus open RP. ${ }^{5}$ Using the SEER-Medicare linked database, this group identified 5556 men who underwent robotic-assisted RP (RARP) and 7878 men who underwent open RP, from 2004 to 2009.

In this cohort, the investigators reported that the incidence of positive surgical margins was significantly lower among men undergoing RARP versus ORP $(13.7 \%$ vs. $18.4 \%$, odds ratio [OR]: $0.68,95 \% \mathrm{Cl} 0.63$ to $0.73, p<0.001)$. The reduction was more pronounced among men with more advanced (intermediate- and high-risk disease).

\section{Denosumab for the prevention of skeletal events}

Dr. Laurence Klotz (Sunnybrook and Women's College Health Sciences Centre, Toronto) was part of an international team of researchers investigating the ability of denosumab to prevent symptomatic skeletal events (SSE, defined as symptomatic fracture, surgery or radiation to bone, or spinal cord compression) in men with CRPC. ${ }^{6}$ The investigators used data from a randomized, controlled trial comparing denosumab to zoledronic acid among men with CRPC and bone metastases. They reported that fewer patients in the denosumab group had confirmed first SSE and multiple SSEs compared to the zoledronic acid group (22\% relative risk reduction $[R R R]$ ).

\section{Denosumab or zoledronic acid therapy on pain interference and cancer- specific QOL (CSQOL)}

Dr. Donald Patrick (University of Washington, Seattle) and his international colleagues presented the results of an ad-hoc analysis of denosumab or zoledronic acid on pain interference and CSQOL.7 The 1901 male patients in this analysis had no prior IV bisphosphonate use. The patients had CRPC and bone metastases and were randomized to receive either
SC denosumab $120 \mathrm{mg}$ plus IV placebo or SC placebo plus IV zoledronic acid $4 \mathrm{mg}$ (adjusted for creatinine clearance) every four weeks. Denosumab therapy delayed the time from baseline to pain increase on overall score (HR 0.83, 0.71 to $0.98, p=0.023$ ), physical (HR $0.87,0.75$ to $1.02, p=0.077$ ) and emotional (HR 0.83, 0.71 to $0.97, p=0.020$ ) subdomains. Overall, treatment with denosumab significantly delayed the length of time before worsening of pain interference. CRPC patients with bone metastases receiving denosumab maintained a higher overall CSQOL compared to those patients receiving zoledronic acid.

\section{Canadian highlights in renal cell cancer research at GU-ASCO 2014}

\section{Positive surgical margins after partial nephrectomy (PN)}

Dr. Rahul Bansal (McMaster University, Hamilton) and colleagues across Canada used the Canadian Kidney Cancer information system (CKCis) database to determine the rate and predictors of positive surgical margins (PSM) after PN for renal cell carcinoma (RCC). ${ }^{8}$

The database included 1066 patients who underwent $\mathrm{PN}$ for RCC in major academic centres across Canada. Among these patients, PSM was reported for 59 individuals (5.5\%). In multivariate analysis, only pathological stage $\geq \mathrm{T} 3$ and Fuhrman grade 4 were predictive of PSM. Age, operative technique, tumour size and tumour stage were not significant independent predictors.

\section{Survival rate with different sunitinib dosing regimens}

Dr. Winnie Cheng and colleagues (British Columbia Cancer Agency [BCCA] - Vancouver Centre) identified 180 patients with RCC in the BCCA database who had been treated with sunitinib from 2007 to $2011 .{ }^{9}$ Their goal was to compare the efficacy and safety of standard intermittent sunitinib therapy with alternative sunitinib dosing regimens. The database included 120 patients receiving standard, intermittent therapy, 32 patients receiving continuous therapy and 28 treated with "nonconventional" regimens (e.g., 2 weeks on therapy/1 week off). For the primary outcome of OS, there was a significant benefit identified for the nonconventional group compared to the intermittent group (median survival 23 months vs. 9 months; HR 0.55, 95\% Cl 0.34 to $0.90, p=0.016$ ). There was no significant difference between the continuous and intermittent groups in OS.

For progression-free survival (PFS), both the continuous and nonconventional groups had significantly longer median durations (median 9 months and 10 months, respectively) compared to intermittent therapy (median 4 months). There is a cur- 
rently a prospective Canadian trial underway investigating the efficacy and safety of individualized sunitinib dosing regimens.

\section{Outcomes of pT3 RCC in Canada}

Dr. Jasmir Nayak (Winnipeg, Manitoba) and Canadian colleagues presented the results of their analysis examining pathological and oncological outcomes for surgically-treated patients with pT3 RCC..$^{10} \mathrm{~A}$ total of 498 patients with pT3 disease were identified in the CKCis database. The majority of these patients $(443[89.0 \%])$ underwent a radical nephrectomy $(\mathrm{RN})$ and 55 (11.0\%) underwent a PN. Of the pT3 lesions, 365 (73.3\%) were pT3a, $97(19.5 \%)$ were pT3b and $12(2.4 \%)$ were pT3c.

A total of 109 patients $(22 \%)$ had diagnosed metastases diagnosis before or at the time of nephrectomy. Of the remaining 389 patients, $68.9 \%$ remained free of disease after a median follow-up of 1.3 years. No peri-operative ( $<30$ days) mortalities were reported, but complications were observed in $14.1 \%$ of patients. Systemic therapy was initiated in $132(26.5 \%)$ of patients, most commonly those who were treated with sunitinib (106 patients).

\section{References}

NB: In addition to the published citations below, information for this article was also taken from the posters and lectures presented at the $2014 \mathrm{GU}-\mathrm{ASCO}$ meeting.

1. Cheng HH et al. The effect of prior abiraterone (Abi) use on the activity of enzalutamide (Enza) in men with mCRPC. J Clin Oncol 2014;32(suppl 4; abstr 18).

2. Azad $A$ et al. Population-based analysis of a novel prognostic model for metastatic castration-resistant prostate cancer (mCRPC) patients (pts) treated with abiraterone acetate (AA). J Clin Oncol 2014;32 (suppl 4; abstr 29).

3. Berlin A et al. Using NBN to predict biochemical relapse following image-guided radiotherapy (IGRT) for intermediate-risk prostate cancer (IR-PCa). J Clin Oncol 2014;32 (suppl 4; abstr 26).

4. Abdollah $\mathrm{F}$ et al. Using adjuvant radiotherapy to improve cancer-specific survival in patients with highly aggressive prostate cancer: Examining recently released criteria. J Clin Oncol 2014;32(suppl 4; abstr 30).

5. Hu JC et al. Population-based comparison of surgical margin status for robotic versus open radical prostatectomy. J Clin Oncol 2014;32(suppl 4; abstr 51).

6. Smith MR et al. Denosumab for the prevention of symptomatic skeletal events in patients with castration-resistant advanced prostate cancer: A comparison with skeletal-related events. J Clin Oncol 2014;32 (suppl 4; abstr 35).

7. Patrick $D$ et al. Denosumab or zoledronic acid (ZA) therapy on pain interference and cancer-specific quality of life (CSQoL) in patients with castrate-resistant prostate cancer (CRPC) and bone metastases (BM). J Clin Oncol 2014;32(suppl 4; abstr 12).

8. Bansal RK et al. Positive surgical margins after partial nephrectomy for renal cell carcinoma: Results from Canadian Kidney Cancer Information System database. J Clin Oncol 2014;32(suppl 4; abstr 420).

9. Cheng $\mathrm{W}$ et al. Survival outcomes associated with different sunitinib dosing regimens in metastatic renal cell carcinoma. J Clin Oncol 2014;32(suppl 4; abstr 417).

10. Nayak I et al. Contemporary outcomes of pT3 renal cell carcinoma: A Canadian multi-institutional experience. J Clin Oncol 2014;32(suppl 4; abstr 434).

Correspondence: Dr. Anil Kapoor, Professor of Surgery (Urology) and Chair, Genitourinary Oncology Program, Juravinski Cancer Centre, McMaster University, St. Joseph's Hospital, 50 Charlton Ave. E., Hamilton 0N L8N 4A6; akapoor@mcmaster.ca 